\title{
Frequency Sampling for Radiative Transfer Calculations in Cool Stars
}

\section{CHRISTIANE HELLING ${ }^{1,2}$ and UFFE GRÅE JØRGENSEN ${ }^{1}$}

${ }^{1}$ Niels Bohr Institute, University Observatory, Copenhagen, Denmark

${ }^{2}$ Institut für Astronomie und Astrophysik, Technische Universität Berlin, Germany

A central problem in combining existing hydrostatic computations for photospheres of cool stars with existing hydrodynamic codes for computation of the wind and circumstellar environment is the thorough optimisation of the frequency selection for the radiative transfer problem. Most hydrostatic models are today based on the Opacity Sampling (OS) method, where the monochromatic opacity in a few thousand frequency points are used for solving the radiative transfer problem. In contrast, solution of the hydrodynamic problem is so much more CPU-demanding that existing codes have to rely on a much more simplified treatment of the radiative transfer. Typically, the radiative transfer in such codes is based on a single value of the opacity - for example the Rosseland mean opacity, or even simpler approximations.

We have analysed the effect of different choices of Opacity Sampling frequencies on hydrostatic photospheric models and have compared the results with those obtained by use of the Rosseland mean or constant-value opacities frequently applied in hydrostatic models. We find that considerable improvement over the results from such mean values can be obtained with Opacity Sampling in as few as 20 frequency points. In the upper photospheric layers of cool carbon-rich giants, typical temperature deviations between a detailed OStreated model (with 5300 frequency points) and corresponding models based on absorption coefficients approximated by 1) OS with 500 points, 2) OS with 50 points, 3) OS with 20 points, 4) a constant-value of $10^{-4} \mathrm{~cm}^{2} / \mathrm{g}$, and 5) the Rosseland mean, were found to be $50 \mathrm{~K}, 100 \mathrm{~K}, 200 \mathrm{~K}, 700 \mathrm{~K}$ and $700 \mathrm{~K}$, respectively. The Rosseland mean models approach the detailed models deep in the atmosphere, whereas the constant-value models deviate very much from the detailed models in deep layers.

Details of our analysis, and a corresponding analysis of oxygen-rich stars, are described in the Diploma Thesis of C. Helling (July 1996) and by Helling \& Jørgensen (1998, $A \mathcal{E} A$, in press). We acknowledge support from the Danish Natural Science Research Council. 